\title{
AAOMS Stage 2 and Some Stage 3 Medication-Related Osteonecrosis of the Jaws (MRONJ) Can Be Managed Successfully and Predictably in OMFS Private Practice with Surgical Intervention and Long-term Intravenous Antibiotics
}

\author{
Hung Le, DDS, MD ${ }^{1^{*}}$ and $M$ Anthony Pogrel, DDS, MD, FACS, FRCS \\ ${ }^{1}$ Oral \& Maxillofacial Surgeon, Private Practice, Anaheim, California, USA \\ ${ }^{2}$ Professor, Department of Oral and Maxillofacial Surgery, University of California, San Francisco, USA
}

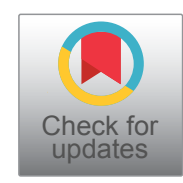

*Corresponding author: Hung Le, DDS, MD, Pacifica Oral \& Maxillofacial Surgery, 408 South Beach Boulevard, Suite 107, Anaheim, California 92804, USA, Tel: (714)-720-0964, Fax: (714)-220-0974

\begin{abstract}
Introduction: Despite published guidelines, the management of medication-related osteonecrosis of the jaws (MRONJ) remains controversial. Most cases that are resistant to straight forward conservative management are usually referred to an academic teaching center or hospitalbased practice. This article will attempt to show that these cases can be managed successfully in private practice on an outpatient basis.

Material and methods: The patients referred to a private practice oral and maxillofacial surgeon with stage 2 and stage 3 MRONJ in the years 2017 and 2020 were included in this study. This project received IRB approval from Pearl IBR, number 21-PACl-101.

Results: Six patients are presented with stage 2 or stage 3 MRONJ who were managed successfully in private practice on an outpatient basis.

Conclusions: It would appear that most cases at stage 2 MRONJ and some cases at stage 3 MRONJ can be managed successfully in private OMFS practice with appropriate outpatient consultations.
\end{abstract}

\section{Introduction}

Medication-related osteonecrosis of the jaws (MRONJ) is a progressive death of jawbone, present for longer than eight weeks in patients who have been treated with anti-resorptive or anti-angiogenic agents in the absence of previous radiation therapy. However, there is debate over the appropriate treatment strategy with lack of consensus among treatment guidelines, particularly in an international setting. The American Association of Oral and Maxillofacial Surgeons (AAOMS) position paper of 2014 [1] and the Japanese position paper from 2017 [2] state that no surgical treatment is recommended for stages 0 and 1 and most stage 2 MRONJ. However, they do state that mobile bony sequestra should be removed to facilitate soft tissue healing. Surgical debridement or resection is only indicated for stage 3 MRONJ. Antibiotic therapy is recommended for stages 1-3 according to the AAOMS guidelines. However, there is no specific recommendation about the type of antibiotic, duration of treatment, or routes of administration. The AAOMS position paper does not mention longterm intravenous antibiotics. Most oral and maxillofacial surgeons in private practice in the USA (and many other countries worldwide) follow the AAOMS guidelines to treat MRONJ with conservative therapy.

However, it is probably true that in most cases, if the disease process does not resolve uneventfully, most patients are referred to an academic or hospital-based oral and maxillofacial surgery service.

The authors will present cases to demonstrate

Citation: Le H, Pogrel MA (2021) AAOMS Stage 2 and Some Stage 3 Medication-Related Osteonecrosis of the Jaws (MRONJ) Can Be Managed Successfully and Predictably in OMFS Private Practice with Surgical Intervention and Long-term Intravenous Antibiotics. Res Rep Oral Maxillofac Surg 5:056. doi. org/10.23937/2643-3907/1710056

Accepted: August 14, 2020; Published: August 16, 2021

Copyright: (C) $2021 \mathrm{Le} \mathrm{H}$, et al. This is an open-access article distributed under the terms of the Creative Commons Attribution License, which permits unrestricted use, distribution, and reproduction in any medium, provided the original author and source are credited. 
that aggressive surgical debridement with tensionless primary closure and longterm intravenous antibiotic result in a predictable outcome for stage 2 and stage 3 MRONJ in both osteoporotic and cancer patients in an outpatient private practice setting without the need for hospitalization but with appropriate outpatient consultations.

\section{Materials and Methods}

All cases of stage 2 and 3 MRONJ referred to a single private practitioner of oral and maxillofacial surgery $(\mathrm{HL})$ during the period January 1, 2017 to December 31, 2020 were included in this study. The underlying pathology, and medications received were recorded as well as all treatments provided. Patients were categorized according to the AAOMS position statement [1].

\section{Stage 0 disease}

Patient with no clinical evidence of exposed bone but with non-specific symptoms or abnormal clinical and radiographic findings.

\section{Stage 1 disease}

Exposed and necrotic bone or fistulae that probe to bone in patients who are asymptomatic and have no evidence of infection. These patients may also present with radiographic findings mentioned for Stage 0 which are localized to the alveolar bone region.

\section{Stage 2 disease}

Exposed and necrotic bone or fistulae that probe to bone with evidence of infection. These patients are typically symptomatic. These patients may also present with radiographic findings mentioned for Stage 0 which are localized to the alveolar bone region.

\section{Stage 3 disease}

Exposed and necrotic bone, or fistulae that probe to bone, with evidence of infection and one or more of the following:

a) Exposed necrotic bone extending beyond the region of alveolar bone, i.e., inferior border and ramus in the mandible, maxillary sinus and zygoma in the maxilla.

b) Pathological fracture.

c) Extraoral fistula.

d) Oroantral/oronasal communication.

e) Osteolysis extending to the inferior border of the mandible or sinus floor.

\section{Results}

Six patients were identified with Stage 2 or Stage 3 MRONJ who were referred to the private practice oral and maxillofacial surgeon ( $\mathrm{HL}$ ) during the study period. There were one male and five females. The age ranged from 72 to 83 with a mean of 78.5. Four patients had received oral bisphosphonates in the form of alendronate (Fosamax) and two received intravenous bisphosphonates. Five patients received the therapy for osteoporosis, and one received the therapy for malignant disease. Four patients presented with Stage 2 disease and two patients presented with Stage 3 disease. Microbiology was performed on all lesions and all grew normal oral flora, whilst in addition two grew Actinomycosis. All six patients had failed conservative treatment with oral antibiotics and pain medications. One patient had failed treatment with surgical debridement and oral antibiotics.

\section{Case Studies}

\section{Case 1}

This was an 83-year-old male who had stage IV multiple myeloma. He had been treated with chemotherapy and 10 infusions of zoledronate (Zometa) three years prior to referral. He was referred by his general dentist for infection of the right mandible. Examination showed necrotic bone in the right maxilla and an intraoral fistula with purulent discharge in the right mandible (Figure 1a). He also had purulent discharge from the gingival sulcus around every tooth. Panoramic radiograph showed a radiolucency in the right maxilla and mandible (Figure 1b). The patient had been treated conservatively for three years with chlorhexidine mouthwash and oral amoxicillin. During this time the infection was under control. However, the necrotic bone in the right maxilla remained unhealed and the right mandibular lesion continued to discharge. He then developed Clostridium Difficile colitis and the amoxicillin was discontinued following which the infection quickly recurred. At this time, his cancer also relapsed, and his general health deteriorated. His chemotherapy was repeated and when his health was improved, surgical debridement and long-term IV antibiotics were proposed. Due to a low hemoglobin $(9 \mathrm{mg} / \mathrm{dL})$ and low platelet count (60k), the patient received two units of packed red blood cell, as an outpatient, prior to debridement. A peripherally inserted central catheter (PICC) line was placed prior to the surgery and monitored anesthesia care was managed by a separate anesthesiologist in the private practitioner's freestanding office. Microbiology specimens were collected and sent to Quest Diagnostics Laboratories. Two teeth were removed and sequestrectomy was performed on the mandible. Following this, sequestrectomy was performed on the right maxilla where an oroantral defect was closed with a buccal fat pad and a buccal mucosa flap. Specimens were sent for pathological examination. Intravenous ertapenem was commenced immediately after the surgery as an empiric antibiotic following outpatient consultation with an infectious disease specialist. He was maintained with nil by mouth for two weeks postoperatively and was given total parenteral nutrition 

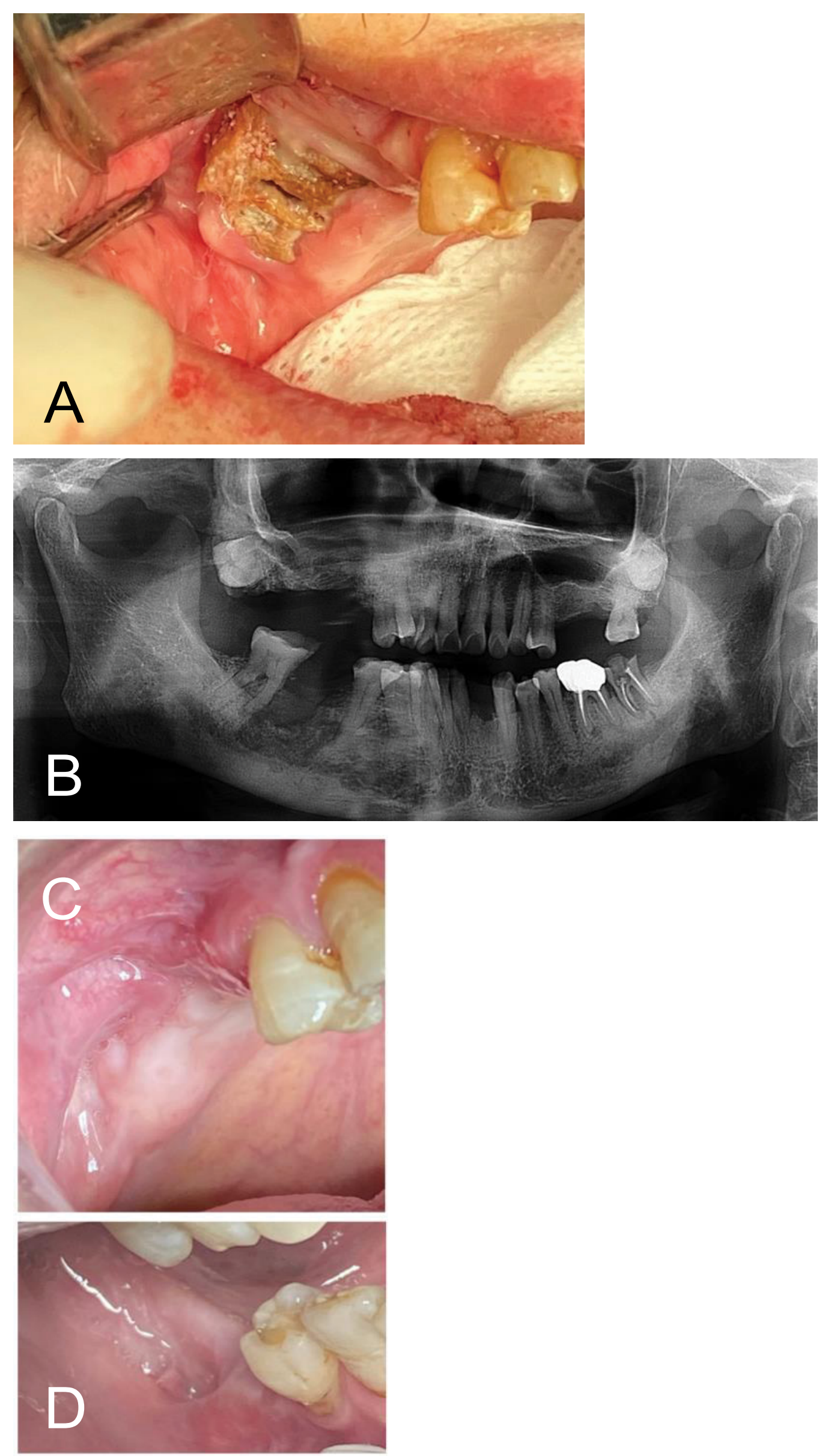

Figure 1: (a) Necrotic bone exposure in right maxilla after ten infusions of Zometa; (b) Panoramic radiograph showing radiolucency of right maxilla and mandible; (c) Wounds in maxilla and mandible six-month after the treatment showing complete epithelialization. 
at home following consultation with appropriate specialists. Microbiology reported normal oral flora sensitive to most antibiotics. Ceftriaxone was added to the ertapenem one week postoperatively. After a six-week intravenous antibiotic course, he was given three further weeks on oral penicillin VK. The patient is currently doing well with no exposed bone, and infection free for almost one year following completion of treatment (Figure $1 \mathrm{c}$ and Figure $1 \mathrm{~d}$ ).

\section{Case \#2}

An 82-year-old female had osteoporosis which was treated with zoledronate in the form of Reclast for two years (one $5 \mathrm{mg}$ infusion per year) following which she developed pain and swelling in the anterior mandible. Her lower anterior teeth were mobile with a yellowish purulent discharge from the lesion (Figure 2a). Six mobile anterior teeth were removed, and the lesion was surgically debrided all of the way to sound bleeding bone. Specimens were sent for oral pathology and sulfur granules typical for actinomycosis was found in the pathology specimens. The patient was first treated with oral amoxicillin for three weeks, but the infection was not under control. Surgical debridement was repeated again in the private practice office and the patient received ertapenem infusion through a PICC line for eight weeks. The patient is now fully healed and has been infection free for two years (Figure $2 b$ ).

\section{Case \#3}

A 72-year-old Filipina female with a history of osteoporosis was treated with oral alendronate in form of Fosamax. She had been taking this regularly (35 mg once weekly) for over ten years. Exposed necrotic bone appeared in the right maxilla and left mandible with purulent discharge three weeks following tooth extraction carried out by her general dentist. The infection was treated with oral amoxicillin for two years before she was referred to an oral and maxillofacial surgeon ( $\mathrm{HL})$. The patient reported that every time she
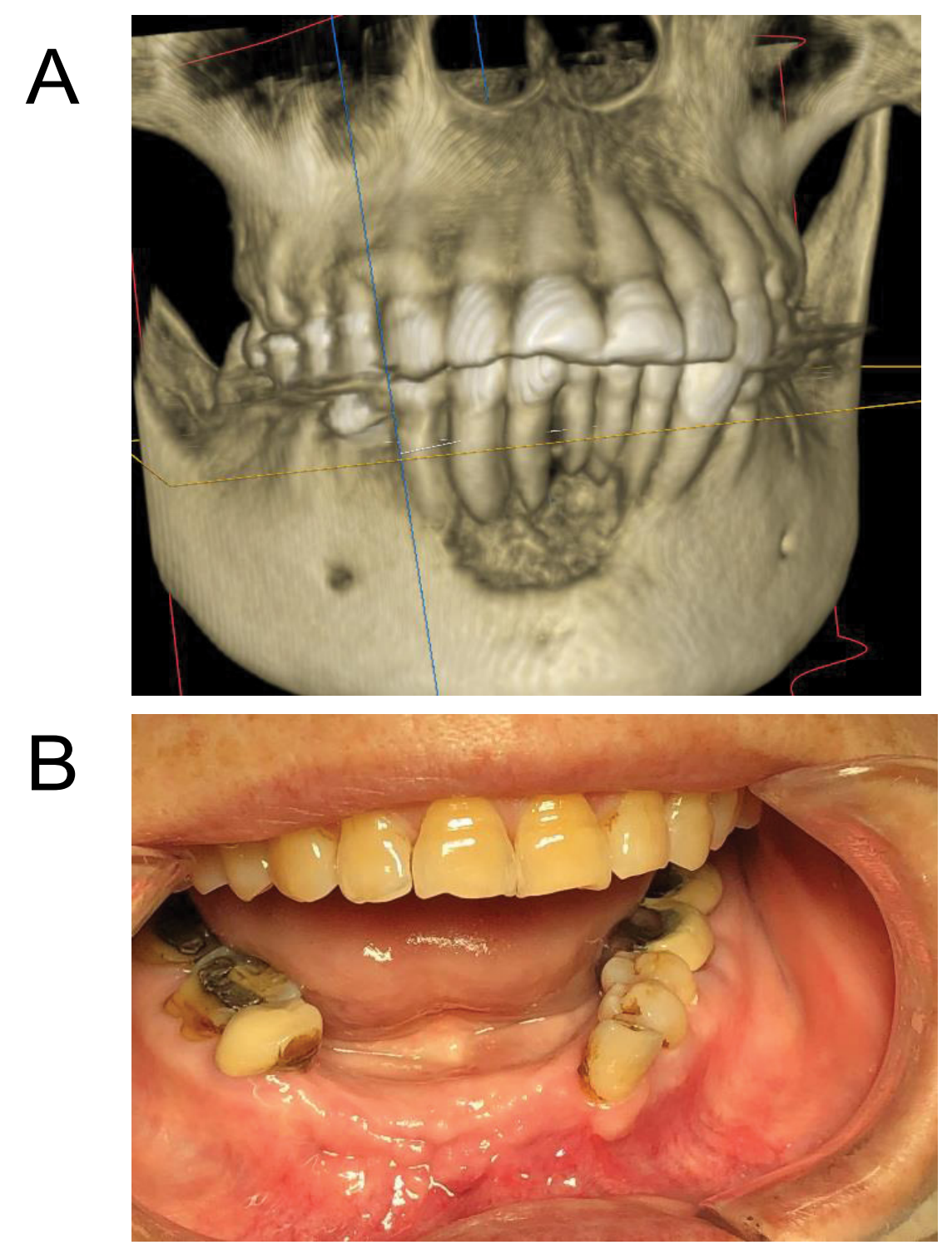

Figure 2: (a) Defect in anterior mandible, illustrated on 3D Cone Beam CT scan; (b) Anterior mandibular wound eight-months postop showing infection free. 

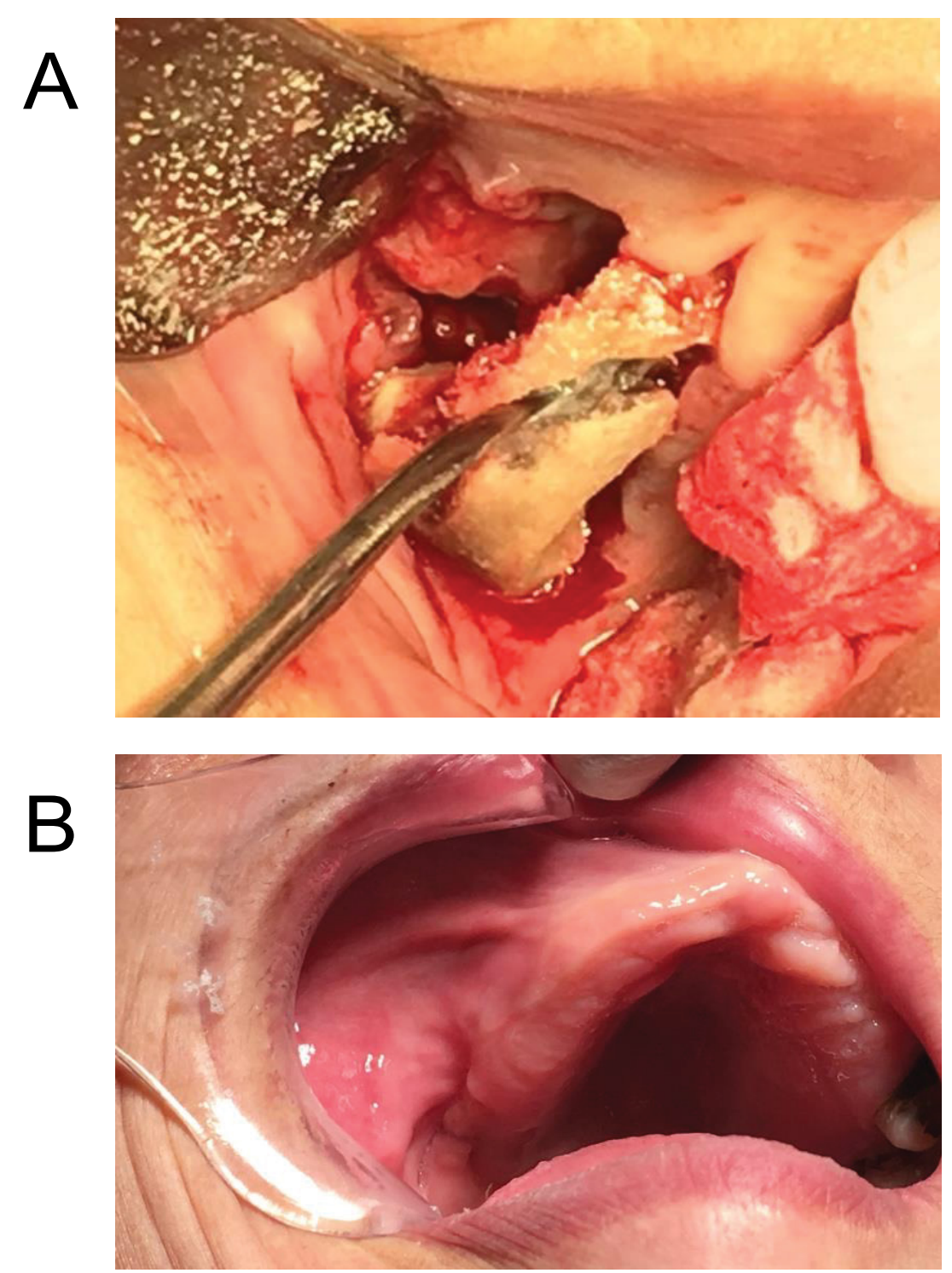

Figure 3: (a) Necrotic bone being removed en-bloc from right maxilla; (b) Right maxilla wound had healed well at fourmonth postop follow-up visit.

came off the antibiotics, the purulent discharge rapidly recurred. The patient underwent surgical debridement with primary closure in the office and six weeks of IV ertapenem. The infection resolved, the mucosa healed, and the patient has been infection and pain free for three years (Figure $3 a$ and Figure $3 b$ ).

\section{Discussion}

Bisphosphonates and other antiresorptive agents are commonly used to treat osteoporosis and bone metastases from solid tumors and also multiple myeloma. They act primarily by inhibiting osteoclast function and secondarily by inhibiting osteoblast function [3]. In this way, they significantly reduce or inhibit bone turnover. They also act on soft tissues inhibiting endothelial cell function with a reduction in angiogenesis [4]. However, they do run the risk of causing MRONJ amongst a number of possible complications. The risk of MRONJ varies considerably with the medication used, the route of administration and the length of time for which it is administered [4]. Zoledronate appears to be associated with the greatest risk of MRONJ which may be as much as $6.7 \%$ for patients who have had infusions of this medication for one year or longer [5], whilst the risk with oral alendronate may be less than $0.1 \%$, although this may increase after the patient has taken the medication for three years or longer [6]. The majority of cases of MRONJ occur following a dental intervention, most often a dental extraction where healing does not occur, and it progresses to MRONJ [7]. Necrotic bone cannot revitalize, may interfere with soft tissue healing, and can be become colonized with bacteria. Treatment should therefore be aimed at removal of necrotic bone together with administration of the appropriate antibiotics.

In the six cases identified for this paper, microbiology was carried out in the submucosal areas and on the sequestra removed in all cases. The organisms isolated, Streptococcus spp, Prevotella spp, and Veillonella spp, present in high frequency in MRONJ along with 
Actinomyces spp [8]. All of these organisms are sensitive to most of the common antibiotics that might be used in these circumstances, but the effectiveness of the antibiotics may vary depending on how they are administered. It does appear that in the treatment of MRONJ, parenteral antibiotics are more effective than oral antibiotics, particularly when coupled with surgical debridement of any necrotic bone.

Beta-lactams are the most commonly prescribed antibiotics in any specialty [9]. They include the penicillins, cephalosporins, mono-bactams, and carbapenems, which all share a common beta-lactam ring binding to penicillin-binding proteins on the bacterial cell wall and inactivating them. Carbapenems such as ertapenem, meropenem, imipenem, and doripenem are the most effective broad-spectrum antibiotic drugs against gram-positive, gram-negative, anaerobic bacteria and Actinomyces spp which are often found in MRONJ lesions. Ertapenem has a once-a-day dosing schedule which allows for convenient outpatient intravenous treatment and was therefore the antibiotic favored for this series of patients, though carbapenem resistance has the potential to become a problem [10].

At the present time, most stage 3 MRONJ patients are not treated in private practice by oral and maxillofacial surgeons, but are referred to an academic or hospital-based oral and maxillofacial surgery service and treatment is commonly carried out in an operating room. Since many of these patients are elderly and in poor general health, it would be beneficial if they could be treated locally and even on an outpatient basis, so they did not need to travel long distances and spend time in hospital. This paper attempts to show that it is possible to treat stage 2 and many stage 3 cases of MRONJ in private practice providing one uses appropriate outpatient subspecialty consultation services and commercial or academic laboratories. PICC lines can be installed in an outpatient setting and Total Parenteral Nutrition, if required, can also be given in an outpatient setting, as can feeding via a nasogastric tube or a percutaneous endoscopic gastrotomy tube.

\section{Conclusion}

As physicians and dentists become more familiar with the etiology, prevention and management of MRONJ, it does appear that the incidence of this disease and also the severity may be decreasing, but even so, many cases of stage 2 and stage 3 disease still occur. This paper attempts to show that the majority of these cases can be treated satisfactorily in a private oral and maxillofacial surgery office with all the advantages of convenience and cost effectiveness that this brings with it. Aggressive surgical debridement, long-term intravenous antibiotics and team approach are keys for successful management of stage 2 and many stage 3 MRONJ cases. One must be able to consult with appropriate specialist colleagues and also have appropriate laboratory facilities available. Postoperative nil by mouth might be necessary for patients with larger defects. For the more complex surgical procedures, it may be advantageous to have a second person responsible for the anesthesia so that the oral and maxillofacial surgeon can concentrate on the surgery.

\section{References}

1. Ruggiero SL, Dodson TB, Fantasia J, Gooday R, Agaloo T, et al. (2014) American Association of Oral and Maxillofacial Surgeons position paper on medication-related osteonecrosis of the jaw-2014 update. J Oral Maxillofac Surg 72: 1938-1956.

2. YonedaT, Hagino $H$, Sugimoto $T$, Ohta $H$, Takahashi $S$, et al. (2017) antirersorbative agent-related osteonecrosis of the jaw. Position Paper 2017 of the Japanese Allied committee on Osteonecrosis of the Jaw. J Bone Miner Metab 35: 6-19.

3. Allen MR, Burr DB (2009) The pathogenesis of bisphosphonate-related osteonecrosis of the jaw: So many hypotheses, so few data. J Oral Maxillofac Surg 67: 61-70.

4. Wood J, Bonjean K, Ruetz S, Bellahcene A, Devy L, et al. (2002) Novel antiangionenic effects of the bisphosphonate compound zoledronic acid. J Pharmacol Exp Ther 302: 1055-1061.

5. Coleman R, Woodward E, Brown J, Cameron D, Bell R, et al. (2011) Safety of zoledronic acid and incidence of osteonecrosis of the jaw (ONJ) during adjuvant therapy in a randomized phase III trial (AZURE: BIG 01-04) for women with stage II/III breast cancer. Breast Cancer Res Treat 127: 429-438.

6. Lo JC, O'Ryan FS, Gordon NP, Yang J, Hui RL, et al. (2010) Prevalence of osteonecrosis of the jaw in patients with oral bisphosphonate exposure. J Oral Maxillofac Surg 68: 243-253.

7. Saad F, Brown JE, Van Poznak C, Ibrahim T, Stemmer SM, et al. (2012) Incidence, risk factors, and outcomes of osteonecrosis of the jaw: integrated analysis from three blinded active-controlled phase III trials in cancer patients with bone metastases. Ann Oncol 23: 1341-1347.

8. Zirk M, Wenxel C, Buller J, Zoller JE, Zinser M, et al. (2019) Microbial diversity in infections of patients with medicationrelated osteonecrosis of the jaw. Clin Oral Investig 23: 2143-2151.

9. Klein EY, Van Boeckel TP, Martinez EM, Pant S, Gandra S, et al. (2018) Global increase and geographic convergence in antibiotic consumption between 2000 and 2015. Proc Natl Acad Sci USA 115: E3463-E3470.

10. Meletis G (2016) Carbapenem resistance: Overview of the problem and future perspectives. Ther Adv Infect Dis 3: 1521. 